\title{
Financial Markets: Very Noisy Information Processing
}

\author{
MALIK MAGDON-ISMAIL, ALEXANDER NICHOLSON, AND YASER S. ABU-MOSTAFA
}

\author{
Invited Paper
}

\begin{abstract}
We report new results about the impact of noise on information processing with application to financial markets. These results quantify the tradeoff between the amount of data and the noise level in the data. They also provide estimates for the performance of a learning system in terms of the noise level. We use these results to derive a method for detecting the change in market volatility from period to period. We successfully apply these results to the four major foreign exchange $(F X)$ markets. The results hold for linear as well as nonlinear learning models and algorithms and for different noise models.
\end{abstract}

Keywords-Bounds, convergence, generalization error, learning, model limitation, noise, test error, volatility.

\section{INTRODUCTION}

Information processing of financial data entails the extraction of relevant information from overwhelming noise. The levels of noise in financial markets are such that the most one can hope for is "getting it right" slightly better than $50 \%$ of the time [1]. To complicate matters further, one also needs to be reasonably sure that one is not being fooled by a finite set of examples from historical data into believing that the performance is acceptable when it is actually (and disastrously) slightly worse than acceptable.

In addition to being a nuisance that complicates the processing of financial data, noise plays a role as a tradable commodity in its own right. Indeed, market volatility is the basis for a number of financial instruments, such as options [2], whose price explicitly depends on the level of volatility in the underlying market. For this reason, it is of economic value to be able to predict the changes in the noise level in financial time series as these changes are reflected in the price changes in tradable instruments. These changes can be significant, as one can observe in Fig. 1, where the

Manuscript received November 1, 1997; revised April 17, 1998. This work was supported in part by the Center for Neuromorphic Systems Engineering (a National Science Foundation supported Engineering Research Center) under National Science Foundation Cooperative Agreement EEC 9402726.

The authors are with Learning Systems Group, California Institute of Technology, Pasadena, CA 91125 USA (e-mail: malik@work.caltech.edu; zander@work.caltech.edu; yaser@caltech.edu).

Publisher Item Identifier S 0018-9219(98)07859-1.
U.S. dollar/German mark market has undergone extreme changes in volatility.

In spite of the high levels of noise, financial data are among the best application domains for intelligent processing and advanced learning techniques. These data have been recorded very accurately for very long periods of time. They are available on different time scales and are simultaneously available in many different markets. This provides a very rich environment for analysis and experimentation using advanced processing techniques. Moreover, the payoff for even minute, but consistent, improvements in performance is huge.

In this paper we tackle the question of information processing of financial data and how it is affected by the presence and variability of noise in the data. In doing so, we do not restrict the distribution or the time-varying nature of the noise, nor do we restrict the learning model or learning algorithm that we use. We report new results that provide quantitative estimates of the optimal performance that can be achieved in the presence of noise. In financial markets, this provides a benchmark for the target performance given a set of data. We also quantify the tradeoff between the amount of data needed and the level of noise in the data. Our experiments with real foreign exchange (FX) data demonstrate that the results are applicable to the case of finite data, which is the only case of practical interest. They also provide a means of assessing the change in the level of noise in financial data that can be applied to volatility-based financial instruments.

The paper is organized as follows. Section II introduces financial time series and Section III covers the main results about the impact of noise. These results are tested in the four major FX markets in Section IV. The appendixes include the formal definitions, theorems, and complete proofs of all the results that we report.

\section{Financial Time Series Prediction}

Financial markets present us with data in the form of a time series. We might have the daily, hourly, or tick-by-tick 


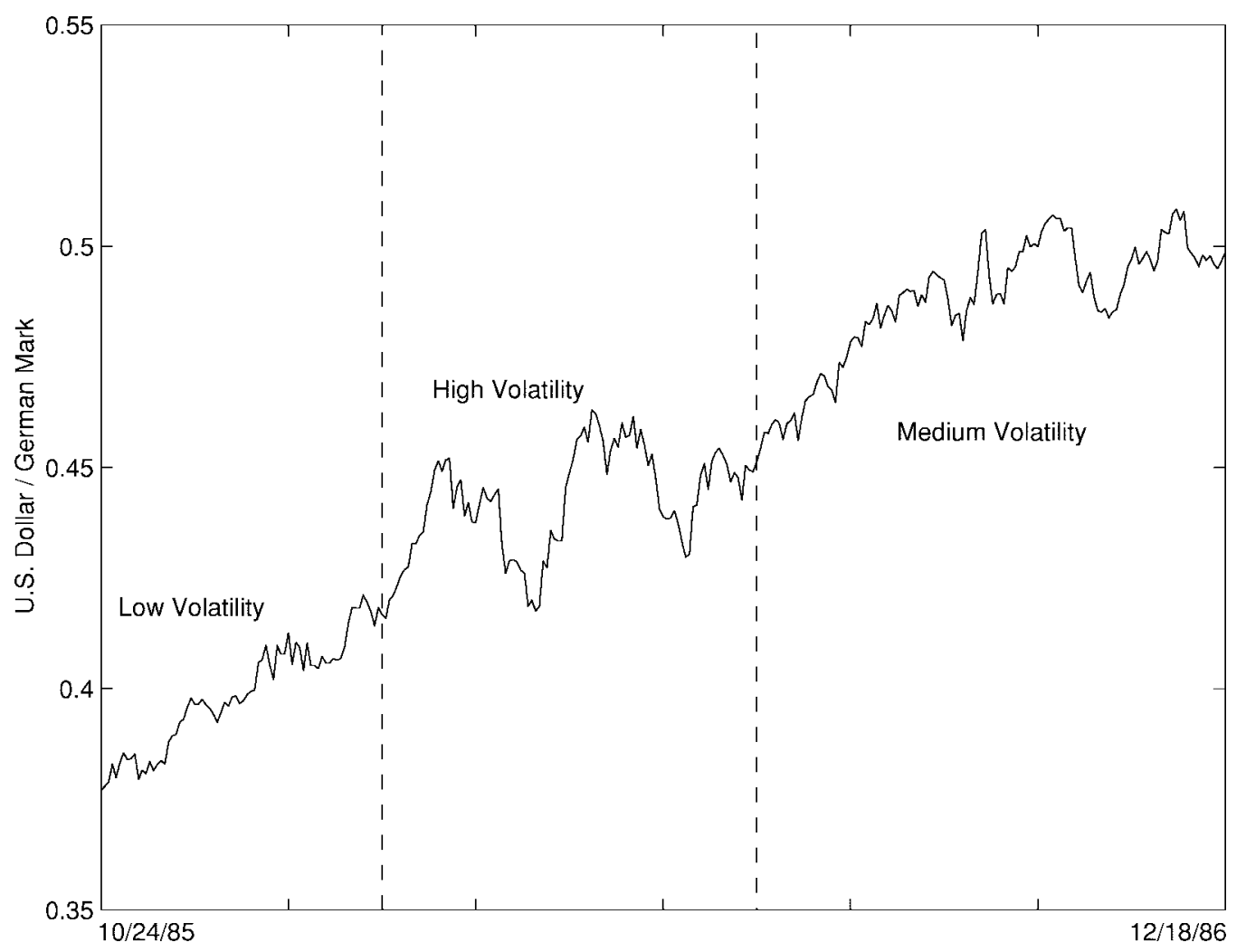

Fig. 1. The price curve for the U.S. dollar versus the German mark. The market volatility can change noticeably over the course of time.

stock prices or FX rates. For financial time series, it is of economic interest to predict the value at some time in the future. Thus, we would like to extract as much information as possible from historical data with the hope of learning the underlying behavior. In general, we can consider the value of a time series $y(t)$ at any time $t$ as a noisy data point $y=f(\mathrm{x})+\epsilon$. Here $f$ is a deterministic function of a vector $\mathbf{x}(t)$ of market indicators and $\epsilon(t)$ is noise (we illustrate this in Fig. 2). The task at hand is one of learning $f(\cdot)$ from a finite data set (the history of the series).

In modeling the time variation of a stock price $S$, the standard Black-Scholes model for pricing options based on volatility [2] assumes the variation to be of the form

$$
d S=\tilde{\mu} S d t+\tilde{\sigma} S \eta \sqrt{d t}
$$

where $\tilde{\sigma}$ is the market volatility and $\eta$ has a zero-mean normal distribution with variance one. Thus, the Black-Scholes model uses only the previous price as the indicator vector $\mathrm{x}$. The price at different times has a deterministic dependence on the past (the $\tilde{\mu}$ term) and a noisy component (the $\tilde{\sigma}$ term). The variance of the noisy component is related to the volatility and need not be constant. The precise relation is given by

$$
\sigma_{i}^{2}=\tilde{\sigma}^{2} \Delta S_{i}^{2}
$$

where $i$ is a time index, and $\Delta S_{i}$ is increment in the stock price from time $i$ to time $i+1$.

Extensive literature already exists on methods for extracting information from noisy time series [3]-[7]. The details of such methods are not our present concern. We are interested in determining how our prediction performance depends on the amount of available data and the variability of the data [which is related to market volatility (1)] - what change in performance are we to expect if this year's market is more volatile than last years market? What change in performance relative to some benchmark are we to expect if the market changed recently and hence we only have a few data points from which to learn?

Pricing information is available on a variety of time scales, which presents us with a data set size versus variability tradeoff. We could choose to use the tick-bytick data because we will then have many data points, but the price we have to pay is that these data points are much noisier. The tradeoff will depend on how much noisier the tick-by-tick data is and the details of the learning scheme. Market analysts would like to quantify this tradeoff by how it affects performance.

An estimate of the best performance that we can achieve with a given information extraction scheme might also be economically useful. In addition to providing a criterion for selecting between different models, knowing the model limitation could be useful for determining whether even an unlimited amount of data will give a system that is financially worth the risk. This would allow analysts to compare trading strategies based on their model limitation.

It is to be expected that when markets are volatile, the performance of a learning system drops. However, the effects of the noise should become less pronounced with 


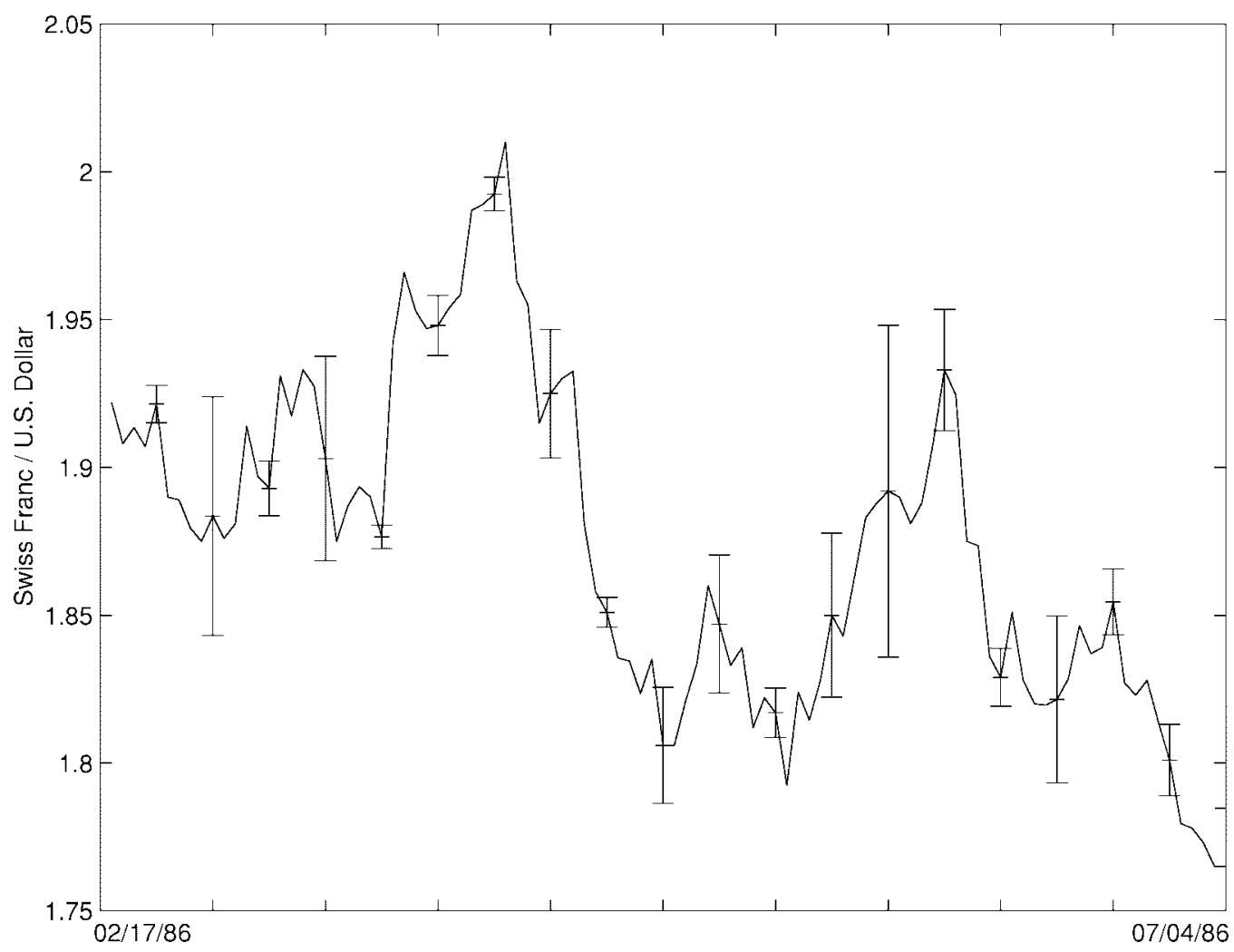

Fig. 2. Financial time series tend to be very volatile. Above is the realization of one such time series from the FX markets. Error bars are used merely to illustrate that each point is the outcome of a noisy event.

increasing data availability. In the next section we quantify this intuition.

\section{IMPACT OF NOISE ON LEARNING}

In this section we address the issues raised in Section II in the context of learning theory. We begin by setting up the learning problem, restate the questions in the learning theory framework, and present theoretical and experimental results.

\section{A. The Learning Problem}

We assume the standard learning paradigm. The goal is to learn a target function $f: \mathbf{R}^{d} \rightarrow \mathbf{R}$. A training data set $\mathcal{D}_{N}$ is given, which consists of $N$ input output pairs $\left\{\mathbf{x}_{i}, y_{i}\right\}_{i=1}^{N}$. Each $\mathbf{x}_{i} \in \mathbf{R}^{d}$ is drawn from some input probability measure $d F(\mathrm{x})$ which we assume to have compact support. Learning entails choosing a hypothesis function $g$ from a collection of candidate functions $\mathcal{H}$. We will assume that the target function $f$ and the candidate functions $g \in \mathcal{H}$ are continuous. The set $\mathcal{H}$ is called the learning model because it reflects how we choose to model the target function. The hypothesis function is chosen by a learning algorithm $\mathcal{A}$ based on some performance criterion on the data. We assume that $\mathcal{A}$ is a mapping $\mathcal{A}: \mathcal{D}_{N} \rightarrow \mathcal{H}$. A typical learning algorithm might be one that uses gradient descent to select the hypothesis which minimizes the mean squared error on the training set. Given a learning task, we

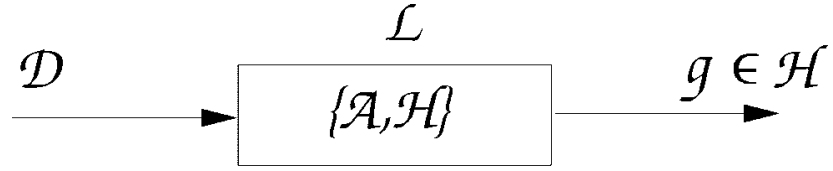

Fig. 3. The learning setup.

select a particular learning system, which takes a data set as input and produces a hypothesis function (see Fig. 3).

Definition III.1: A learning system $\mathcal{L}$ is a pair $\{\mathcal{A}, \mathcal{H}\}$. Additive noise is present in the training data

$$
y_{i}=f\left(\mathbf{x}_{i}\right)+\epsilon_{i} \text {. }
$$

We further assume that the noise realizations are independent and zero mean, so

$$
\langle\epsilon \mid \mathbf{x}\rangle_{\epsilon}=\mathbf{0}, \quad\left\langle\epsilon \epsilon^{T} \mid \mathbf{x}\right\rangle_{\epsilon}=\operatorname{diag}\left[\sigma_{1}^{2}, \sigma_{2}^{2}, \ldots, \sigma_{N}^{2}\right]
$$

(we use $\langle\cdot\rangle$ to denote expectations, $\sigma=\left[\sigma_{1} \sigma_{2} \ldots \sigma_{N}\right]$ and $\operatorname{diag}[\cdot]$ denotes a diagonal matrix). It should be noted that we allow the noise variance to change from one data point to another, which is always the case in financial markets.

Define $g_{\mathcal{D}_{N}}(\mathrm{x}) \in \mathcal{H}$ as $\mathcal{A}\left(\mathcal{D}_{N}\right)$, the function that was chosen by the algorithm. We define the test error for $g_{\mathcal{D}_{N}}$ as the expectation of the squared deviation between $g_{\mathcal{D}_{N}}$ and $f(\mathrm{x})$ taken over the input space. Thus the test error measures the ultimate performance of our system after it has learned from the data. We denote the test error by $E\left[g_{\mathcal{D}_{N}}\right]$ as follows:

$$
E\left[g_{\mathcal{D}_{N}}\right]=\left\langle\left(g_{\mathcal{D}_{N}}(\mathbf{x})-f(\mathbf{x})\right)^{2}\right\rangle_{\mathbf{x}}
$$




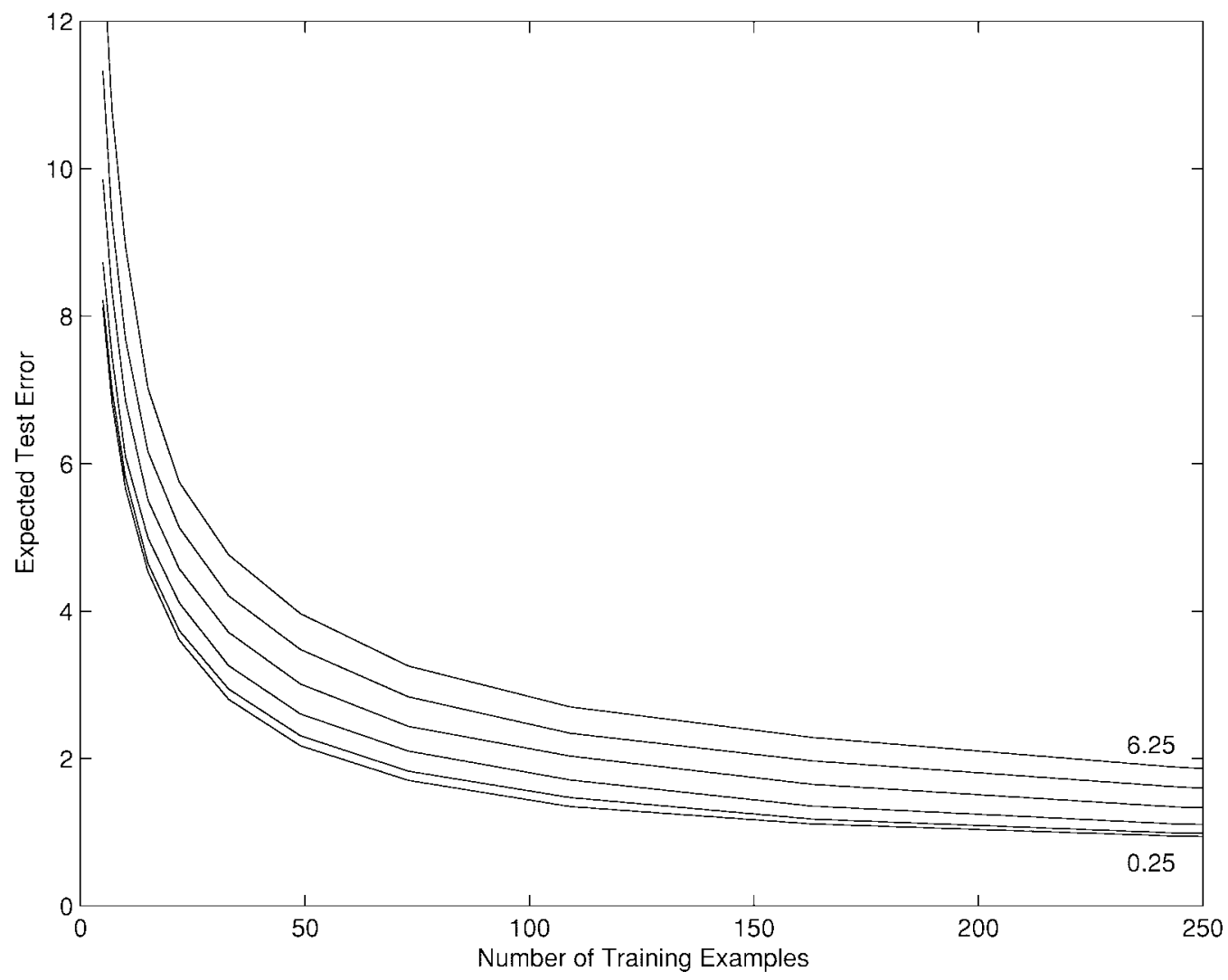

Fig. 4. Experiments illustrating the behavior of the test error as a function of $N$ for various noise levels with variances ranging from $0.25-6.25$. A nonlinear neural network learning model was used with gradient descent on the squared error. Data was created using a nonlinear target function.

We can further define the expected test error $\mathcal{E}_{N}(\sigma)$ as the expectation of the test error taken over possible realizations of the noise and the data set as follows:

$$
\mathcal{E}_{N}(\sigma)=\left\langle E\left[g_{\mathcal{D}_{N}}\right]\right\rangle_{\epsilon, \mathcal{D}_{N}}
$$

The goal is to minimize $\mathcal{E}_{N}(\sigma)$. $\mathcal{E}_{N}(\sigma)$ represents the expected test performance averaged over the choice of training examples. It is related to the "future profit" you expect to make having trained your learning system on the available data. $\mathcal{E}_{N}(\sigma)$ will depend on the detailed properties of the learning system and target function. It would be a daunting task to tackle the behavior of $\mathcal{E}_{N}(\sigma)$ in general, but as we shall see, under quite unrestrictive conditions the changes in $\mathcal{E}_{N}(\sigma)$ as the noise or data set size change can be quantified. This will be related to the tradeoff in profit when attempting to learn and predict during more volatile stages of the market compared to less volatile stages.

A related quantity of interest is $\mathcal{N}$, the number of data points (with noise added) that are needed to attain a test error comparable to that attainable when $N$ noiseless examples are available.

$$
\mathcal{N}(\Delta, \sigma, N) \triangleq \min _{N_{1}}\left\{N_{1}: \mathcal{E}_{N_{1}}(\sigma)-\mathcal{E}_{N}(0) \leq \Delta\right\}
$$

$\mathcal{N}(\Delta, \sigma, N)$ is the number of noisy examples that are equivalent to $N$ noiseless examples, and it describes the tradeoff between numerous, more volatile data versus fewer and less volatile data. The answers to the questions posed in Section II lie in the behavior of $\mathcal{E}_{N}(\sigma)$ and $\mathcal{N}(\Delta, \sigma, N)$. We address these questions analytically next.

\section{B. Performance of a Learning System}

Intuition tells us that noisier data leads to worse test performance. This is because the learning system attempts to fit the noise (i.e., learn a random effect) at the expense of fitting the true underlying dependence. However, the more data we have, the less pronounced the impact of the noise will be. This intuition is illustrated in Fig. 4. We observe that the higher the noise, the higher the test error, but the curves appear to be getting closer to each other as we use more and more examples for the learning process. We would like to quantify this idea.

In order to be able to do so, we need to restrict ourselves to stable learning systems. Stable learning systems possess the two properties "continuity" and "unbiasedness." Continuity ensures that "close" data sets are mapped to "close" functions. For two data sets differing only by the addition of zero-mean noise, unbiasedness requires that, at every point, the average value (with respect to the noise) of the functions resulting from the noisy data set is equal to the value of the function resulting from the noiseless data set. (Refer to the appendixes for formal definitions.)

These properties are somewhat intuitive, and we note that, for any learning system $\mathcal{L}$, they can be checked directly. We would like our learning procedure to be robust toward small noise fluctuations in the data so we do not 
consider learning models that may yield discontinuous behavior. The unbiasedness property may seem fragile, especially given the extremely nonlinear nature of a learning algorithm. Nevertheless, we consider it an important and not overly restrictive condition on a learning system. If the noise is small, then the first order change in $\mathcal{A}\left(D_{N}\right)$ should be proportional to the noise parameter so that the average change is zero with zero-mean noise. Indeed, experiments with neural networks show that learning with gradient descent and conjugate gradient descent on the mean squared error are unbiased with a reasonable noise level. Thus, linear and neural network learning models give learning systems that are stable.

We then have the following theorem.

Theorem III.2: Let $\mathcal{L}$ be stable. Then $\forall \epsilon>0, \exists \mathcal{C}_{1}$ such that using $\mathcal{L}$, it is at least possible to attain a test error bounded by

$$
\begin{aligned}
& \mathcal{E}_{N}(\sigma)<\mathcal{E}_{N}(0)+\frac{\overline{\sigma^{2}} \mathcal{C}_{1}}{N}+\epsilon+O\left(\frac{1}{N^{2}}\right) \\
& \mathcal{E}_{N}(0)<E_{0}+\frac{\mathcal{C}_{2}}{N}+\epsilon+o\left(\frac{1}{N}\right)
\end{aligned}
$$

where $\lim _{N \rightarrow \infty} \mathcal{E}_{N}(0)=E_{0}$ and $\overline{\sigma^{2}}=\frac{1}{N} \sum_{i=1}^{N} \sigma_{i}^{2} \cdot \mathcal{C}_{1}, \mathcal{C}_{2}$ are constants that depend on the input distribution, target function, and learning system.

The proof can be found in Appendix II (Theorem B.5). Furthermore, in certain cases we can combine (5) and (6) to get

$$
\mathcal{E}_{N}(0)<E_{0}+\frac{\mathcal{C}_{1} \overline{\sigma^{2}}+\mathcal{C}_{2}}{N}+o\left(\frac{1}{N}\right) .
$$

The essential content of the theorem is that the expected test error increases in proportion to $\overline{\sigma^{2}}$ holding everything else constant, and it decreases in proportion to $1 / N$ holding everything else constant. The conditions of Theorem III.2 are quite general and are satisfied by a wide variety of learning models and algorithms. For learning models that are linear $\mathcal{C}_{1}=d+1$. $E_{0}$ is the model limitation modulo the learning algorithm when tested on noiseless data. The limiting performance on noisy future data is $E_{0}+\overline{\sigma^{2}}$. One expects that for more complex models, the model limitation $\left(E_{0}\right)$ is lower than for less complex learning models. However, the convergence parameters $\left(\mathcal{C}_{1}, \mathcal{C}_{2}\right)$ are expected to be larger for more complex models. Thus, for a given number of data points, there will be an optimal model complexity (e.g., number of hidden units for a neural network) minimizing the bound of Theorem III.2. One can compare this tradeoff to the bias-variance tradeoff [8].

Experimentally, we observe that the bounds of Theorem III.2 are quite tight even for small $N$ (see Fig. 5), so combining (5) and (6) we expect the following dependence for $\mathcal{N}(\Delta, \sigma, N)$, the number of noisy examples that are equivalent to $N$ noiseless examples:

$$
\mathcal{N}(\Delta, \sigma, N) \sim \frac{\overline{\sigma^{2}} \mathcal{C}_{1}+\mathcal{C}_{2}}{\frac{\mathcal{C}_{2}}{N}+\Delta} .
$$

The results are illustrated in Fig. 5. Artificial data sets were created from a known target function. Fig. 5(a) illustrates the results of fitting a linear model to nonlinear data. Shown is the residual error $\hat{\mathcal{E}}_{N}(\sigma)=\mathcal{E}_{N}(\sigma)-$ $\mathcal{E}_{N}(0)$. The inputs are chosen from $\mathbf{R}^{2}$, and the dashed lines illustrate that $\hat{\mathcal{E}}_{N}(\sigma)$ quickly converges to $3 \overline{\sigma^{2}} / N$ as expected from (5). Fig. 5(b) shows similar results for a nonlinear learning model. Gradient descent was used to train the three hidden unit neural network models. Ideally, we expect this algorithm/model pair to be continuously compatible, and it was empirically shown to be mean preserving. The residual errors very closely follow $20 \overline{\sigma^{2}} / \mathrm{N}$, showing that we have approximate equality in (5) for $C_{1} \sim 20 .{ }^{1}$ Figs. 5(c) and (d) show that $\mathcal{E}_{N}(0)$ also behaves linearly in $1 / N$ for both cases [i.e., it quickly approaches the bound in (6)].

\section{Estimating the Model Limitation}

When the learning model is linear, we can show (Theorems B.2 and B.3) that the expected training error $\mathcal{E}_{\mathrm{tr}}(\sigma)$ (the error on the data set) and expected test error approach the same limiting value from opposite sides as $N \rightarrow \infty$. Furthermore, the rates of convergence to this limiting value are the same. Amari [9] has obtained a similar asymptotic result in the case of nonlinear models when performing gradient descent on the training error. Using the Amari result, we can use our bound on the test error to bound the training error performance. The expected error on a noisy data set $\mathcal{E}_{\text {test }}$ is related to $\mathcal{E}_{N}(\sigma)$ by $\mathcal{E}_{\text {test }}(\sigma)=\mathcal{E}_{N}(\sigma)+\overline{\sigma^{2}}$. The experiments demonstrate that the bounds of Theorem III.2 are almost saturated for small $N$, so, ignoring terms that are $o(1 / N)$, and using Amari's result we have

$$
\begin{aligned}
& E_{0}+\overline{\sigma^{2}} \leq \mathcal{E}_{\text {test }}(\sigma) \approx E_{0}+\overline{\sigma^{2}}+\frac{\mathcal{C}_{1} \overline{\sigma^{2}}+\mathcal{C}_{2}}{N} \\
& E_{0}+\overline{\sigma^{2}} \geq \mathcal{E}_{\operatorname{tr}}(\sigma) \approx E_{0}+\overline{\sigma^{2}}-\frac{\mathcal{C}_{1} \overline{\sigma^{2}}+\mathcal{C}_{2}}{N}
\end{aligned}
$$

(in the case of linear learning models we can replace $\mathcal{C}_{1}$ by $d+1)$. From the data set of size $N$, for $N_{1}<N$, we can randomly pick $N_{1}$ data points (perform bootstrapping [10] on the training data). Thus, by varying $N_{1}$ in the training phase and observing the error on the training set, we obtain an estimate of the model limitation $E_{0}+\overline{\sigma^{2}}$. This method also immediately furnishes an estimate of $\mathcal{C}_{1} \overline{\sigma^{2}}+\mathcal{C}_{2}$, so we can estimate the parameters that are needed for the bound (7). This is illustrated in the Section IV, where we apply the results presented here to the case of financial time series.

\section{APPliCATION to FinANCIAL MARKET ForeCASTING}

We can apply the results of Section III to real financial market data. Fig. 6 illustrates the $1 / N$ behavior of the residual error $\hat{\mathcal{E}}_{N}(\sigma)$ for $\mathrm{FX}$ rates.

Daily close exchange rates between 1984 and 1995 were used for the Swiss franc (CHF), German mark (DEM), British pound sterling (STG) and Japanese yen (JPY). A

\footnotetext{
${ }^{1}$ This suggests that the condition in Corollary B.6 holds.
} 


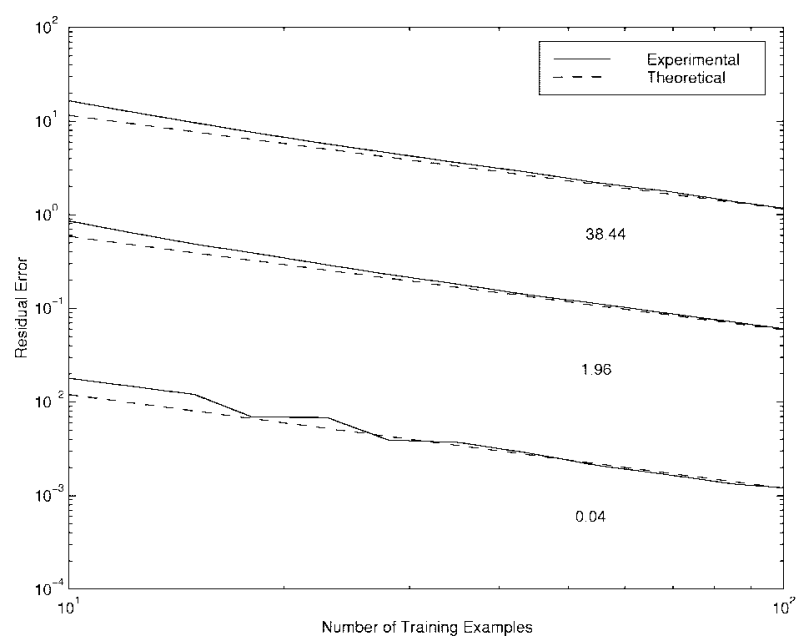

(a)

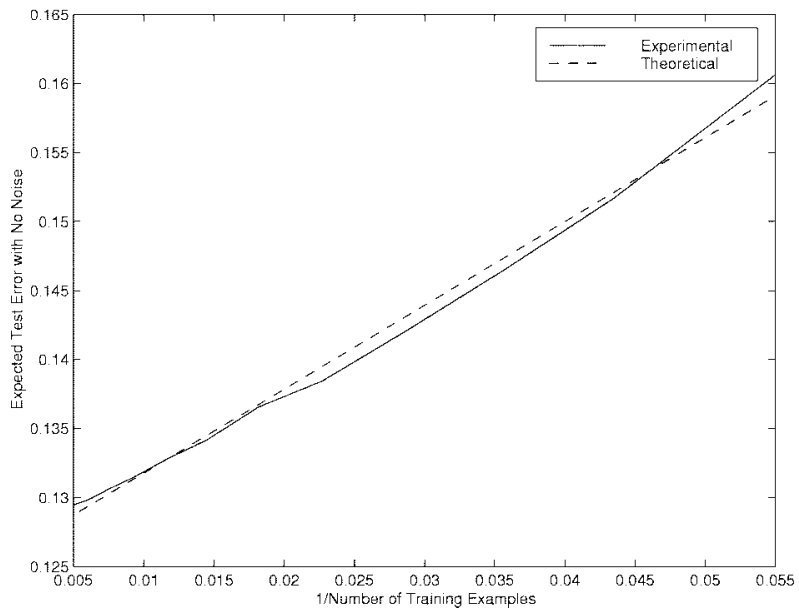

(c)

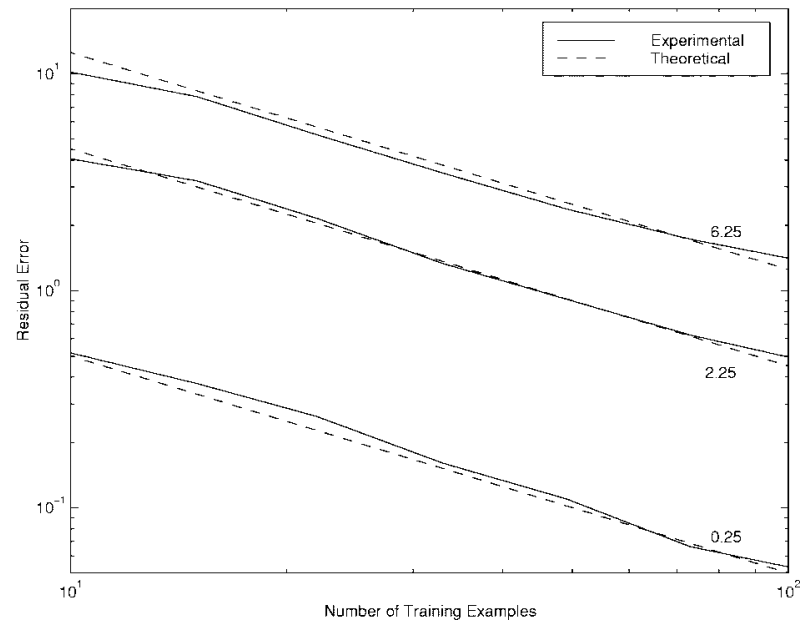

(b)

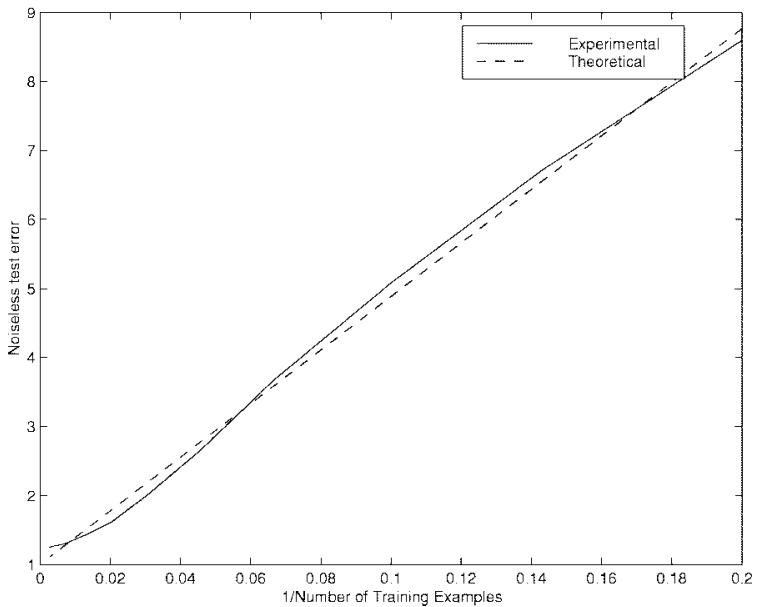

(d)

Fig. 5. (a) Nonlinear target function and linear learning model - the residual error is shown for a nonlinear target function trained using a linear model. Gaussian noise with $\overline{\sigma^{2}}$ ranging from 0.04 to 38.44 was added to training sets. The dashed lines show the error level predicted by Theorem B.2. (b) Nonlinear target function and nonlinear learning model-the residual error is shown for a nonlinear target function trained using a 2-3-1 network. Gaussian noise with $\overline{\sigma^{2}}$ ranging from 0.25 to 6.25 was added to training sets. The results correspond very closely to those predicted by Theorem III. 2 when $C_{1}=20$ (shown with dashed lines). (c) The behavior of the expected test error with no noise for the learning scenario in (a). We observe that for even small $N$ there is close agreement between the theoretical $1 / N$ decrease. (d) The behavior of the expected test error with no noise for the nonlinear learning scenario in (b). Once again for small $N$ we observe the expected behavior.

linear model was used to learn the future price as a function of the close price of the previous five days.

We performed the following experiments. The last 1000 data points of each time series were held out as a test set. The remaining points were used to create a data set

$$
\left\{\mathbf{x}_{k}=\left(S_{k-4}, \ldots, S_{k}\right), y_{k}=S_{k+1}\right\} \text {. }
$$

$N_{1}$ points were sampled from this set and used to learn. This was repeated to obtain an estimate of the expected test and training error. We show the dependence of the expected test error on the number of training examples in Fig. 6. Though it is not obvious that the assumptions made to derive the results hold, as with the results on artificial data, the test error seems to not only obey the bound of (5), but it assumes quickly $1 / N$ behavior. Assuming the bounds to be tight for both the test error and training error, we are able to estimate the best possible performance of the linear model by finding the line best fitting $\mathcal{E}_{N}(0)$ as a function of $1 / N$. Table 1 summarizes these estimates.

We compare the model limitation to that of simply predicting the present value as the next value. We find that this simple strategy virtually attains the model limitation suggesting that today's price completely reflects tomorrow's price-which is the best we can expect to achieve systematically. The results in Table 1 are appealing on two accounts. First, assuming that today's price is the best predictor of tomorrow's price, the technique we use to predict the model limitation is performing well. Second, because the model limitation estimates are slightly below the error of the simple strategy, we deduce that 


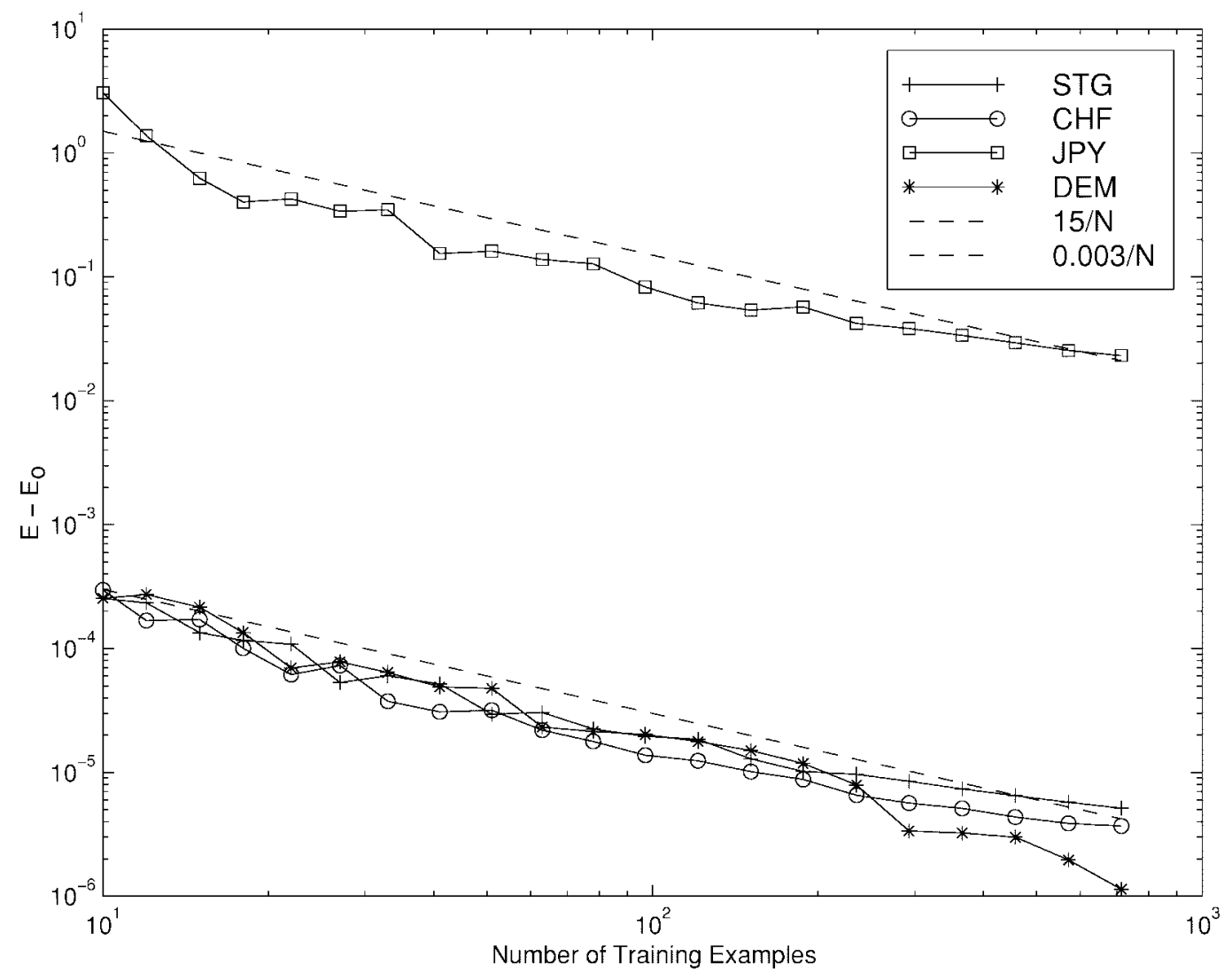

Fig. 6. The dependence of the test error- $E_{0}$ on $N$ is depicted for the British pound (STG), the Swiss franc (CHF), the Japanese yen (JPY), and the German mark (DEM). Also shown are two lines that show $1 / N$ behavior. We see that the test error curves follow the theory well.

Table 1 Estimate Of Model Limitation and Comparison to Simple Predictor ${ }^{2}$

\begin{tabular}{c|c|c}
\hline Currency & $E_{0}$ Est. & No Change \\
\hline DEM & 0.000499 & 0.000502 \\
\hline CHF & 0.000158 & 0.000160 \\
\hline STG & 0.000134 & 0.000136 \\
\hline JPY & 1.082 & 1.083 \\
\hline
\end{tabular}

(a)

\begin{tabular}{c|c|c}
\hline Currency & $E_{0}$ Est. & No Change \\
\hline DEM & 0.000156 & 0.000152 \\
\hline CHF & 0.000148 & 0.000151 \\
\hline STG & 0.000153 & 0.000157 \\
\hline JPY & 0.851 & 0.867 \\
\hline
\end{tabular}

(b)

there is some information that can be extracted from previous prices.

By training on different time periods, we find that the model limitation may change. If we assume the underlying dependence to have remained constant so that $E_{0}$ has not

\footnotetext{
${ }^{2}$ In (a) we use the training error to estimate $E_{0}+\overline{\sigma^{2}}$ and compare to the performance on the training set when we use the simple system "predict no change in price." In (b) we use the test error curve to estimate $E_{0}$. Only (a) is possible in practice, but both yield very good estimates (if we assume that this simple strategy is close to the best you can do), thus verifying that the results of Section III can be applied to this learning problem. The change in the estimate from (a) to (b) is due to the fact that the test and training sets are taken from different time intervals, and hence the estimates reflect a change in the market volatility over time.
}

changed, then the resulting change can only be due to a change in $\overline{\sigma^{2}}$, thus providing an estimate of the change in the volatility [since the volatility is related to the change in $\overline{\sigma^{2}}$ (1)]. It appears from Table 1 that of the four currencies, the STG's volatility seems to have increased while the remaining three markets display decreasing volatility.

We see that the results of Section III apply to the problem of financial forecasting. Experiment bears out the fact that the answers to the questions posed in Section II lie in the expressions for $\mathcal{E}_{N}(\sigma)$ and $\mathcal{N}(\Delta, \sigma, N)$ in (5) and (8).

\section{CONCLUSION}

The new results in Section II are represented in Theorem III.2. The experimental results on artificial data amply support the theory. We have shown that the number of noisy examples required for comparable generalization with $N$ noiseless examples increases as $\overline{\sigma^{2}}$. Explicitly, the main result bounds the test error for a noisy data set by $\mathcal{E}_{N}(\sigma) \leq$ $E_{0}+\left(\overline{\sigma^{2}} \mathcal{C}_{1}^{\prime}+\mathcal{C}_{2}^{\prime}\right) / N+o(1 / N)$. We also obtained a result that bounds the expected test error relative to a benchmark test error (5). Experimentally we showed that this result applies to the nonasymptotic regime-the empirical results show that the bounds hold with almost equality for $N$ as small as 20. Intuitively, this is because the nonasymptotic effects that affect $\mathcal{E}_{N}(\sigma)$ also have a similar effect on $\mathcal{E}_{N}(0)$. 
We began with the goal of answering two questions (initially posed in the context of financial time series): relative to a benchmark scenario (that of learning with no noise), how does the performance change as the noise and number of examples changes? This dependence is represented by the expression for $\mathcal{E}_{N}(\sigma)$ above. This expression is a similar result to those derived by Amari in [9] and Moody in [11]. However, the differences are significant. Amari compares the training error when descending on a given error function to the expectation of that error when you have finished learning. The learning algorithm is specific but the form of the error function may vary. Moody considers minimization of an error term plus a complexity term and assumes that the input distribution is a sum of delta functions at the training data points. In this paper, we derive a convergence result for the expected squared error without severely restricting the learning algorithm or the input distribution. The results were presented in the context of financial time series analysis, but we note that they are applicable to the general learning problem, independent of most of the details of the learning model and learning algorithm. In particular, we do not require the learning algorithm to minimize a simple training error measure-optimizing a generalized regularized training error (as in [12]) should produce an algorithm that still satisfies the conditions of Theorem III.2.

We provided an estimate of the model limitation which we used to estimate the best possible performance when learning in the FX markets. The results were consistent with the assumption that today's price reflects all the information about tomorrow's price. Using this method for predicting the model limitation, we could detect changes in the market volatility, which is of economic use.

It would be useful to explore the relationship between the constants $\left(E_{0}, \mathcal{C}_{1}, \mathcal{C}_{2}\right)$ that parameterize the expected test error dependence.

\section{APPENDIX I}

\section{DEFINITIONS}

One expects that if one has "close" data sets $\mathcal{D}_{N}=$ $\left\{\mathbf{x}_{i}, f\left(\mathbf{x}_{i}\right)\right\}$ and $\mathcal{D}_{N}^{\prime}=\left\{\mathbf{x}_{i}, f\left(\mathbf{x}_{i}\right)+e\left(\mathbf{x}_{i}\right)\right\}$ where $e\left(\mathbf{x}_{i}\right)$ is small, then $\mathcal{A}\left(\mathcal{D}_{N}\right)$ should be "close" to $\mathcal{A}\left(\mathcal{D}_{N}^{\prime}\right)$. For $\mathcal{A}$ to have this property, $\mathcal{H}$ should be able to implement the two "close" functions. ${ }^{3}$ We formalize this notion by defining the class of learning systems that are $n$th order-continuously compatible $\left(\mathcal{C C}_{n}\right)$ with respect to the probability measure $d F(\mathbf{x})$. We will use the following notation. Let $\mathcal{S}$ be the compact support for $d F(\mathbf{x})$ and let $\left\{\mathbf{x}_{i}\right\}_{i=1}^{N} \subset \mathcal{S}$. Let $\mathcal{D}=\left\{\mathbf{x}_{i}, y_{i}\right\}_{i=1}^{N}, \mathcal{D}^{\prime}=\left\{\mathbf{x}_{i}, y_{i}+\epsilon_{i}\right\}_{i=1}^{N}$ be any two data sets on $\mathcal{S}$ such that $\max _{i} \epsilon_{i}=\epsilon_{\max }$. Let $\mathcal{A}(\mathcal{D})=g(\mathrm{x})$, $\mathcal{A}\left(\mathcal{D}^{\prime}\right)=g(\mathbf{x})+\eta(\mathbf{x})$.

Definition A.1: $\mathcal{L}$ is $n$th order-continuously compatible if $\exists C$ such that

$$
\left\langle|\eta(\mathbf{x})|^{n}\right\rangle_{\mathbf{x}} \leq\left(C \epsilon_{\max }\right)^{n}
$$

with probability one (i.e., for almost every $\mathcal{D}$ ). We will write $\mathcal{L} \in \mathcal{C C}_{n}$.

\footnotetext{
${ }^{3}$ These conditions will often be satisfied in practice.
}

We would like $\mathcal{A}$ to be "unbiased" in the following sense. If we have a data set $\mathcal{D}$ with $\mathcal{A}(D)=g_{0}$ and we add independent, zero mean noise to the targets to get a new data set $\mathcal{D}^{\prime}$, then we would like $\left\langle\mathcal{A}\left(\mathcal{D}^{\prime}\right)\right\rangle_{\text {noise }}=g_{0}$, where this average of functions is taken pointwise. This motivates the following definition.

Definition A.2: Let $\mathcal{D}$ and $\mathcal{D}^{\prime}$ be two data sets related by $\mathbf{y}^{\prime}=\mathbf{y}+\epsilon$, where the $\epsilon_{i}$ 's are independent and zero mean. Then $\mathcal{L}$ is mean preserving or unbiased if $\left\langle\mathcal{A}\left(\mathcal{D}^{\prime}\right)\right\rangle_{\epsilon}=$ $\mathcal{A}(\mathcal{D})$ with probability 1 (i.e. for almost every $\mathcal{D}$ ).

Definition A.3: A learning system $\mathcal{L}$ is stable if it is in $\mathcal{C C}_{2}$ and it is mean preserving.

\section{APPENDIX II}

\section{PROOFS OF RESUlTS}

Proposition B.1: If $\mathcal{L} \in \mathcal{C C}_{n}$, then $\mathcal{L} \in \mathcal{C C}_{m}$ for $m=$ $1 \cdots n$.

Proof: By Jensen's inequality, $\left\langle|f(\mathrm{x})|^{a}\right\rangle_{\mathbf{x}} \leq\langle|f(\mathrm{x})|\rangle_{\mathbf{x}}^{a}$ for $0 \leq a \leq 1$. Letting $f(\mathbf{x})=\eta(\mathbf{x})^{n}$, as in Definition A.1, and $a=m / n$ for $m \leq n$, the proposition now follows because $\mathcal{L} \in \mathcal{C C}_{n}$.

We use the notation

$$
\begin{aligned}
& \mathbf{X}=\left[\begin{array}{llll}
\mathrm{x}_{1} & \mathrm{x}_{2} & \ldots & \mathrm{x}_{N}
\end{array}\right], \quad \mathbf{y}=\mathrm{f}+\epsilon \\
& \Sigma \equiv\left\langle\mathbf{x x}^{T}\right\rangle_{\mathbf{x}}, \quad \mathbf{q}=\langle\mathbf{x} f(\mathbf{x})\rangle_{\mathbf{x}} \text {. }
\end{aligned}
$$

The law of large numbers gives us that $\mathbf{X X}^{T} \rightarrow_{N \rightarrow \infty}$ $N \Sigma$ and $\mathbf{X f} \rightarrow_{N \rightarrow \infty} N\langle\mathbf{x} f(\mathbf{x})\rangle_{\mathbf{x}}$. where we assume that the conditions for this to happen are satisfied.

Theorem B.2: Let $\mathcal{H}$, the learning model, be the set of linear functions $\mathbf{w} \cdot \mathbf{x}+w_{0}$, and let the learning algorithm be minimization of the squared error. Then

$$
\begin{aligned}
& \mathcal{E}_{N}(\sigma)=\mathcal{E}_{N}(0)+\frac{\overline{\sigma^{2}}(d+1)}{N}+O\left(\frac{1}{N^{2}}\right) \\
& \mathcal{E}_{N}(0)=E_{0}+\frac{B}{N}+O\left(\frac{1}{N^{\frac{3}{2}}}\right)
\end{aligned}
$$

where $E_{0}=\lim _{N \rightarrow \infty}\left\{\mathcal{E}_{N}(0)\right\}$ and $B$ is a constant dependent on the input distribution. It follows that $\mathcal{N}(\Delta, \sigma, N)=$ $\left(\overline{\sigma^{2}}(d+1)+B\right) /(\Delta+(B / N))+O\left(1 / N^{\frac{3}{2}}\right)$.

Proof: $g \in \mathcal{L} \Leftrightarrow g(\mathbf{x})=\mathbf{x}^{T} \mathbf{w}$. The least squares estimate of $\mathbf{w}$ is given by

$$
\hat{\mathbf{w}}=\left(\mathbf{X X}^{T}\right)^{-1} \mathbf{X} \mathbf{y}
$$

from which we calculate

$$
\begin{aligned}
\mathcal{E}_{N}(\sigma)= & \left\langle\hat{\mathbf{w}}^{T} \mathbf{x} \mathbf{x}^{T} \hat{\mathbf{w}}-2 \hat{\mathbf{w}}^{T} \mathbf{x} f(\mathbf{x})+f(\mathbf{x})^{2}\right\rangle_{\mathbf{x}, \mathbf{X}, \epsilon} \\
= & \left\langle f^{2}\right\rangle-2\left\langle\mathbf{f}^{T} \mathbf{X}^{T}\left(\mathbf{X X}^{T}\right)^{-1}\right\rangle_{\mathbf{X}} \mathbf{q} \\
& +\left\langle\mathbf{f}^{T} \mathbf{X}^{T}\left(\mathbf{X X}^{T}\right)^{-1} \Sigma\left(\mathbf{X} \mathbf{X}^{T}\right)^{-1} \mathbf{X f}\right\rangle_{\mathbf{X}} \\
& +\left\langle\epsilon^{T} \mathbf{X}^{T}\left(\mathbf{X} \mathbf{X}^{T}\right)^{-1} \Sigma\left(\mathbf{X} \mathbf{X}^{T}\right)^{-1} \mathbf{X} \epsilon\right\rangle_{\mathbf{X}, \epsilon} \\
= & \mathcal{E}_{N}(0)+\sum_{i} \sigma_{i}^{2}\left\langle\left[\mathbf{X}^{T}\left(\mathbf{X X}^{T}\right)^{-1}\right.\right. \\
& \left.\left.\cdot \Sigma\left(\mathbf{X X}^{T}\right)^{-1} \mathbf{X}\right]_{i i}\right\rangle_{\mathbf{X}}
\end{aligned}
$$

where we have used (1) and $\mathbf{q}=\langle\mathbf{x} f(\mathbf{x})\rangle_{\mathbf{x}}$. By the law of large numbers, we note that $\left(\mathbf{X X}^{T}\right)^{-1} \rightarrow_{N \rightarrow \infty} N \Sigma$ and 
$\mathrm{Xf} \rightarrow_{N \rightarrow \infty} N \mathbf{q}$, so we write

$$
\mathbf{X X}^{T}=N \Sigma+\sqrt{N} \mathbf{V}(\mathbf{X}) \quad \mathbf{X} \mathbf{f}=N \mathbf{q}+\sqrt{N} \mathbf{a}(\mathbf{X})
$$

where $\langle\mathbf{V}\rangle_{\mathbf{X}}=\langle\mathbf{a}\rangle_{\mathbf{X}}=\mathbf{0}$ and $\operatorname{Var}(\mathbf{V})$ and $\operatorname{Var}(\mathbf{a})$ are $O(1)$. Using (14) and the identity $(1+\lambda \mathbf{A})^{-1}=1-\lambda \mathbf{A}+$ $\lambda^{2} \mathbf{A}^{\mathbf{2}}+O\left(\lambda^{3}\right)$ we have

$$
\begin{aligned}
& \mathbf{X}^{T}\left(\mathbf{X X}^{T}\right)^{-1} \Sigma\left(\mathbf{X X}^{T}\right)^{-1} \mathbf{X} \\
&= \frac{\mathbf{X}^{T} \Sigma^{-1} \mathbf{X}}{N^{2}}-2 \frac{\mathbf{X}^{T} \Sigma^{-1} \mathbf{V} \Sigma^{-1} \mathbf{X}}{N^{\frac{5}{2}}} \\
&+3 \frac{\mathbf{X}^{T} \Sigma^{-1} \mathbf{V} \Sigma^{-1} \mathbf{V} \Sigma^{-1} \mathbf{X}}{N^{3}}+O\left(\frac{1}{N^{\frac{7}{2}}}\right) .
\end{aligned}
$$

From the definition of $\mathbf{X}$ we find from the first term

$$
\begin{aligned}
{\left[\left\langle\mathbf{X}^{T} \Sigma^{-1} \mathbf{X}\right\rangle\right]_{i i} } & =\left\langle\sum_{k, l} \Sigma_{k l}^{-1}\left(\mathbf{x}_{i}\right)_{k}\left(\mathbf{x}_{i}\right)_{l}\right\rangle \\
& =\left[\sum_{k, l} \Sigma_{k l}^{-1} \Sigma_{k l}\right]_{i i}=1
\end{aligned}
$$

by taking the expectation of the trace of both sides of the equation, the second term can be shown to be of the same order as the third term. So

$$
\mathcal{E}_{N}(\sigma)=\mathcal{E}_{N}(0)+\frac{\sum_{i} \sigma_{i}^{2}}{N^{2}}+O\left(\frac{1}{N^{2}}\right) .
$$

The first part of the theorem now follows. Using similar techniques for $\mathcal{E}_{N}(0)$, we find

$$
\begin{aligned}
\mathcal{E}_{N}(0)= & \left\langle f^{2}\right\rangle-\mathbf{q}^{T} \Sigma^{-1} \mathbf{q}+\overbrace{\frac{\left\langle\mathbf{q}^{T} \Sigma^{-1} \mathbf{V} \Sigma^{-1} \mathbf{q}-\mathbf{a}^{T} \Sigma^{-1} \mathbf{q}\right\rangle}{N^{\frac{1}{2}}}}^{0} \\
& +\frac{1}{N}\left\langle\mathbf{q}^{T} \Sigma^{-1} \mathbf{V} \Sigma^{-1} \mathbf{V} \Sigma^{-1} \mathbf{q}+\mathbf{a}^{T} \Sigma^{-1} \mathbf{a}\right. \\
& \left.-2 \mathbf{a}^{T} \Sigma^{-1} \mathbf{V} \Sigma^{-1} \mathbf{q}\right\rangle+O\left(\frac{1}{N^{\frac{3}{2}}}\right) \\
= & E_{0}+\frac{B}{N}+O\left(\frac{1}{N^{\frac{3}{2}}}\right)
\end{aligned}
$$

with $E_{0}=\left\langle f^{2}\right\rangle-\mathbf{q}^{T} \Sigma^{-1} \mathbf{q}$ and $B$ depending on the input distribution. This gives the $N$ dependence of $\mathcal{E}_{N}(0)$. Finally we have

$$
\mathcal{E}_{\mathcal{N}}(\sigma)-\mathcal{E}_{N}(0)=\frac{\overline{\sigma^{2}}(d+1)+B}{\mathcal{N}}-\frac{B}{N}=\Delta
$$

yielding the functional dependence $\mathcal{N}(\Delta, \sigma, N)$.

This result can immediately be generalized to the case where the learning model is linear in its parameter space. A similar technique can be used to derive a result on the expected mean squared residual itself which we will call $\mathcal{E}_{r}(\sigma)$.
Theorem B.3: Let $\mathcal{H}$, the learning model, be the set of linear functions $\mathbf{w} \cdot \mathbf{x}+w_{0}$, and let the learning algorithm be minimization of the squared error. Then

$$
\begin{aligned}
& \mathcal{E}_{r}(\sigma)=\mathcal{E}_{r}(0)+\overline{\sigma^{2}}-\frac{\overline{\sigma^{2}}(d+1)}{N} \\
& \mathcal{E}_{r}(0)=E_{0}-\frac{B}{N}+O\left(\frac{1}{N^{\frac{3}{2}}}\right)
\end{aligned}
$$

where $E_{0}$ and $B$ are the same constants appearing in Theorem B.2. Thus we find

$$
\mathcal{E}_{N}(\sigma)-\mathcal{E}_{r}(\sigma)=\frac{2\left(\overline{\sigma^{2}}(d+1)+B\right)}{N}+o\left(\frac{1}{N^{2}}\right)
$$

Proof: The residual error is given by

$$
\begin{aligned}
\mathcal{E}_{r}(\sigma)= & \left\langle\frac{1}{N}\left(\hat{\mathbf{X}}^{T} \mathbf{w}-\mathbf{y}\right)^{2}\right\rangle \\
= & \left\langle\frac{1}{N}\left(\left(\mathbf{X}^{T}\left(\mathbf{X X}^{T}\right)^{-1} \mathbf{X}-\mathbf{1}\right) y\right)^{2}\right\rangle \\
= & \frac{1}{N}\left(\left\langle\mathbf{f}^{T} \mathbf{f}\right\rangle-\left\langle f^{T} \mathbf{X}\left(\mathbf{X} \mathbf{X}^{T}\right)^{-1} \mathbf{X} \mathbf{f}\right\rangle+\left\langle\epsilon^{T} \epsilon\right\rangle\right. \\
& \left.-\left\langle\epsilon^{T} \mathbf{X}^{T}\left(\mathbf{X X}^{T}\right)^{-1} \mathbf{X} \epsilon\right\rangle\right) \\
= & \underbrace{\left\langle f^{2}\right\rangle-\frac{\left\langle\mathbf{f}^{T} X^{T}\left(\mathbf{X} \mathbf{X}^{T}\right)^{-1} \mathbf{X} \mathbf{f}\right\rangle}{N}}_{\mathcal{E}_{r}(0)}+\overline{\sigma^{2}}-\frac{\overline{\sigma^{2}}(d+1)}{N}
\end{aligned}
$$

from which the first part of the theorem follows. Using the techniques of Theorem B.2 we find that

$$
\begin{aligned}
\mathcal{E}_{r}(0)= & \left\langle f^{2}\right\rangle-\mathbf{q}^{T} \Sigma^{-1} \mathbf{q} \\
& -\frac{1}{N}\left\langle\mathbf{q}^{T} \Sigma^{-1} \mathbf{V} \Sigma^{-1} \mathbf{V} \Sigma^{-1} \mathbf{q}+\mathbf{a}^{T} \Sigma^{-1} \mathbf{a}\right. \\
& \left.-2 \mathbf{a}^{T} \Sigma^{-1} \mathbf{V} \Sigma^{-1} \mathbf{q}\right\rangle+O\left(\frac{1}{N^{\frac{3}{2}}}\right) .
\end{aligned}
$$

Comparing with Theorem B.2 we have the second part of the theorem.

This result is similar to the results obtained by [9], [11].

We now consider the case of a nonlinear learning model. The following proposition shows that $\lim _{N \rightarrow \infty} \mathcal{A}\left(\mathcal{D}_{N}\right)=$ $g_{\infty}$ is well defined pointwise-i.e., $\forall \epsilon>0, \exists M$ such that if $N>M$ then $\max _{\mathbf{x}}\left|g_{\infty}-\mathcal{A}\left(\mathcal{D}_{N}\right)\right|<\epsilon$. This can be skipped if this fact is self evident or if one wishes to assume convergence and one is merely interested in the rate. It is included here purely for technical completeness.

Proposition B.4: Let $\mathcal{L} \in \mathcal{C C}_{2}$. Then, the limit $\lim _{N \rightarrow \infty}$ $\mathcal{A}\left(\mathcal{D}_{N}\right)=g_{\infty}$ for noiseless data sets is well defined pointwise on sets of nonzero probability-i.e., $\forall \epsilon>0, \exists M$ such that if $N>M$, then $\max _{\mathbf{x}}\left|g_{\infty}-\mathcal{A}\left(\mathcal{D}_{N}\right)\right|<\epsilon$.

Proof: We will sketch the idea of the proof, the details can be filled in using exactly the same techniques as for the Proof to Theorem B.9. First we show that for any 
two infinite data sets, the learned functions are essentially identical. For any infinite data set, as the input support is compact (closed and bounded), any infinitesimal volume of nonzero probability has an infinite number of data points. Consider two such data sets. The means of the targets in this small volume will be equal (by the law of large numbers). Because the target function is continuous on this compact support, the means for the two data sets are arbitrarily close to the true values for each data set (this can be attained by letting the size of the volume be arbitrarily small). By continuous compatibility, these two data sets must both be mapped arbitrarily close to the data set with the means as targets. Therefore they must be mapped arbitrarily close to each other. Thus, we see that $\left\langle\left(g_{1}-g_{2}\right)^{2}\right\rangle$ is less than $\epsilon$ for arbitrary small $\epsilon$ where the two different data sets drawn from the input distribution are mapped to $g_{i}$. So we conclude that $\left\langle\left(g_{1}-g_{2}\right)^{2}\right\rangle=0$, therefore $g_{1}=g_{2}$ with probability one. Thus, any two infinite noiseless data sets are mapped to the same function (as the functions are continuous), which we call $g_{\infty}$.

Finally, consider a data set $\mathcal{D}_{N}$. For $N$ large enough, this data set can be made arbitrarily close to an infinite data set using the argument above. Let $g_{N}=\mathcal{A}\left(\mathcal{D}_{N}\right)$. Therefore $\left\langle\left(g_{N}-g_{\infty}\right)^{2}\right\rangle$ can be made arbitrarily small by choosing $N$ large enough. In other words, $\lim _{N \rightarrow \infty}\left\langle\left(g_{N}-g_{\infty}\right)^{2}\right\rangle=0$, therefore $g_{N}$ converges to $g_{\infty}$ with probability one. Further, because the functions are continuous and the support is compact, this convergence is uniform.

We have just shown that the limit $\mathcal{A}\left(\mathcal{D}_{N}\right)$ exists as $N \rightarrow$ $\infty$. Thus, with noiseless data sets, we have convergence for stable learning systems. We now consider both the rate of convergence and what happens when noise is added.

Theorem B.5: Let $\mathcal{L}$ be stable. Let the target function $f$ be continuous. Let the probability measure on the input space have compact support $\mathcal{X}$. Then $\forall \epsilon>0, \exists C_{1}>0$ such that using $\mathcal{L}$, it is at least possible to attain a test error bounded by

$$
\mathcal{E}_{N}(\sigma)<\mathcal{E}_{N}(0)+\frac{\overline{\sigma^{2}} C_{1}}{N}+\epsilon+O\left(\frac{1}{N^{2}}\right)
$$

Corollary B.6: If $C_{1} \leq C_{1}^{\prime} \forall \epsilon$, then

$$
\mathcal{E}_{N}(\sigma) \leq \mathcal{E}_{N}(0)+\frac{\overline{\sigma^{2}} C_{1}^{\prime}}{N}+\left(\frac{1}{N^{2}}\right)
$$

Corollary B.7: $\lim _{N \rightarrow \infty} \mathcal{E}_{N}(\sigma)=\lim _{N \rightarrow \infty} \mathcal{E}_{N}(0)$, independent of $\sigma$.

Proof: By rescaling, we can assume that the input space $\mathcal{X} \subseteq \mathcal{S}=[0,1]^{d}$. $f$ is continuous, so it is uniformly continuous on the compact set $\mathcal{S}$. Therefore, $\exists \delta_{1}$ such that

$$
\left|\mathbf{x}-\mathrm{x}^{\prime}\right|<\delta_{1} \Rightarrow\left|f(\mathrm{x})-f\left(\mathrm{x}^{\prime}\right)\right|<\delta_{2}
$$

Divide $[0,1]$ into intervals of size $\delta_{1} / \sqrt{d}$. Thus we divide $\mathcal{S}$ into $\left(\sqrt{d} / \delta_{1}\right)^{d}$ cubes. Let $C_{\mathbf{i}} \equiv C_{i_{1}, i_{2} \cdots i_{d}}$ define the cube with lowest coordinates i. Let $N_{\mathbf{i}}$ be the number of data points in $C_{\mathbf{i}}$, and let $\mu_{\mathbf{i}}=\left(1 / N_{\mathbf{i}}\right) \sum_{x_{j} \in C_{\mathbf{i}}} y_{j}$. Let $P_{\mathbf{i}}=\operatorname{Pr}\left\{x \in C_{\mathbf{i}}\right\}$. We only need consider regions where $P_{\mathbf{i}}>0$, as regions with $P_{\mathbf{i}}=0$ are "don't care" regions. The following Lemma is easily obtained by noting that for $\mathrm{x}, \mathrm{x}^{\prime} \in C_{\mathbf{i}},\left|f(\mathrm{x})-f\left(\mathrm{x}^{\prime}\right)\right| \leq \delta_{2}$.

Lemma B.8: Let $\mathrm{x} \in C_{\mathbf{i}}$

$\left|\frac{1}{N_{\mathbf{i}}} \sum_{\mathbf{x}_{j} \in C_{\mathbf{i}}} y_{j}-f(\mathbf{x})\right|=\left|\mu_{\mathbf{i}}-f(\mathbf{x})\right| \leq \delta_{2}+\frac{\left|\sum_{x_{k} \in C_{i}} \epsilon_{k}\right|}{N_{\mathbf{i}}}$.

Construct a new data set by replacing all the $y$ 's in $C_{\mathbf{i}}$ by $\mu_{\mathbf{i}}$, i.e., with no noise the targets would be $f\left(\mathbf{x}_{j}\right)$, and with noise they are $\mu_{\mathbf{i}} . \forall \mathbf{x}_{j} \in C_{\mathbf{i}}$

$$
\begin{aligned}
\mu_{i} & =f\left(\mathbf{x}_{j}\right)+\underbrace{\sum_{\mathbf{x}_{k} \neq \mathbf{x}_{j}} \frac{f\left(\mathbf{x}_{k}\right)-f\left(\mathbf{x}_{j}\right)}{N_{\mathbf{i}}}}_{|\cdot|<\delta_{2}}+\frac{\sum_{x_{k} \in C_{i}} \epsilon_{k}}{N_{\mathbf{i}}} \\
& =f\left(\mathbf{x}_{j}\right)+\eta_{j}+\xi_{j}
\end{aligned}
$$

where $\eta_{j}=\sum_{\mathbf{x}_{k} \neq \mathbf{x}_{j}}\left(f\left(\mathbf{x}_{k}\right)-f\left(\mathbf{x}_{j}\right) / N_{\mathbf{i}}\right)$ and $\xi_{j}=$ $\sum_{x_{k} \in C_{i}} \epsilon_{k} / N_{\mathbf{i}}$. We have that $\left\langle\eta_{j}\right\rangle_{\mathcal{D}_{N}}=0$ and $\left\langle\xi_{j}\right\rangle_{\epsilon}=0$. Let $\mathcal{A}$ map the noiseless data set to $g_{0} \in \mathcal{H}$ and this noisy version of the data set to $g=g_{0}+\eta$. So for the test error we have

$$
\begin{aligned}
\mathcal{E}_{N}(\sigma)= & \left\langle(f-g)^{2}\right\rangle_{\mathcal{D}_{N}, \mathbf{x}, \epsilon} \\
= & \underbrace{\left\langle\left(f-g_{0}\right)^{2}\right\rangle_{\mathcal{D}_{N}, \mathbf{x}, \epsilon}}_{\mathcal{E}_{N}(0)}+\underbrace{2\left\langle\left(f-g_{0}\right)\left(g_{0}-g\right)\right\rangle_{\mathcal{D}_{N}, \mathbf{x}, \epsilon}}_{T_{1}} \\
& +\underbrace{\left\langle\left(g_{0}-g\right)^{2}\right\rangle_{\mathcal{D}_{N}, \mathbf{x}, \epsilon}}_{T_{2}} .
\end{aligned}
$$

We now examine $T_{1}$ and $T_{2}$.

$$
\begin{aligned}
& \left|T_{1}\right|=\left|\left\langle\left(f-g_{0}\right)\left\langle g_{0}-g\right\rangle_{\epsilon}\right\rangle_{\mathcal{D}_{N}, \mathbf{x}}\right| \\
& \stackrel{(\mathbf{a})}{=} \mid\left\langle( f - g _ { 0 } ) \left(\mathcal{A}\left(\left\{\mathbf{x}_{k}, f\left(\mathbf{x}_{k}\right)\right\}\right)\right.\right. \\
& \left.\left.-\mathcal{A}\left(\left\{\mathrm{x}_{k}, f\left(\mathrm{x}_{k}\right)+\eta_{k}\right\}\right)\right)\right\rangle_{\mathcal{D}_{N}, \mathbf{x}} \mid \\
& \leq \mid\left\langlef \left\langle\left(\mathcal{A}\left(\left\{\mathbf{x}_{k}, f\left(\mathbf{x}_{k}\right)\right\}\right)\right.\right.\right. \\
& \left.\left.\left.-\mathcal{A}\left(\left\{\mathrm{x}_{k}, f\left(\mathrm{x}_{k}\right)+\eta_{k}\right\}\right)\right)\right\rangle_{\mathcal{D}_{N}}\right\rangle_{\mathbf{x}} \mid \\
& +\mid\left\langleg _ { 0 } \left(\mathcal{A}\left(\left\{\mathrm{x}_{k}, f\left(\mathrm{x}_{k}\right)\right\}\right)\right.\right. \\
& \left.\left.-\mathcal{A}\left(\left\{\mathrm{x}_{k}, f\left(\mathrm{x}_{k}\right)+\eta_{k}\right\}\right)\right)\right\rangle_{\mathcal{D}_{N}, \mathrm{x}} \mid \\
& \stackrel{\text { (b) }}{\leq}\left\langle\max _{\mathbf{x}}\left|g_{0}\right|\langle| \mathcal{A}\left(\left\{\mathrm{x}_{k}, f\left(\mathrm{x}_{k}\right)\right\}\right)\right. \\
& \left.-\mathcal{A}\left(\left\{\mathrm{x}_{k}, f\left(\mathrm{x}_{k}\right)+\eta_{k}\right\}\right)|\rangle_{\mathbf{x}}\right\rangle_{\mathcal{D}_{N}} \\
& \stackrel{\text { (c) }}{\leq} C \delta_{2}\left\langle\max _{\mathrm{x}}\left|g_{0}\right|\right\rangle_{\mathcal{D}_{N}} \\
& \text { (d) } \\
& \leq c_{1} \delta_{2}
\end{aligned}
$$

where (a) and (b) follow from the mean preserving assumption. (c) from continuous compatibility and (d) because we assume the limit $g_{\infty}$ to exist pointwise. Similarly, for $T_{2}$ 
we get

$$
\begin{aligned}
& \left|T_{2}\right|=\mid\left\langle\left(\mathcal{A}\left(\left\{\mathrm{x}_{k}, f\left(\mathrm{x}_{k}\right)\right\}\right)\right.\right. \\
& \left.\left.-\mathcal{A}\left(\left\{\mathrm{x}_{k}, f\left(\mathrm{x}_{k}\right)+\eta_{k}+\xi_{k}\right\}\right)\right)^{2}\right\rangle_{\mathcal{D}_{N}, \mathrm{x}, \epsilon} \\
& \stackrel{(\mathrm{a})}{\leq} 2 C^{2}\left(\delta_{2}^{2}+\left\langle\sum_{\mathbf{i}} \frac{\sum_{\mathbf{x}_{j}, \mathbf{x}_{k} \in C_{\mathbf{i}}} \epsilon_{j} \epsilon_{k}}{N_{\mathbf{i}}^{2}}\right\rangle_{\mathcal{D}_{N}, \epsilon}\right) \\
& =2 C^{2}\left(\delta_{2}^{2}+\left\langle\sum_{\mathbf{i}} \frac{\bar{\sigma}_{i}^{2}}{N_{\mathbf{i}}}\right\rangle_{\mathcal{D}_{N}, \epsilon}\right) \\
& \stackrel{(\mathrm{b})}{=} 2 C^{2}\left(\delta_{2}^{2}+\overline{\sigma^{2}}\left\langle\sum_{\mathbf{i}} \frac{1}{N_{\mathbf{i}}}\right\rangle_{\mathcal{D}_{N}}\right) \\
& =2 C^{2} \delta_{2}^{2}+2 C^{2} \overline{\sigma^{2}} \sum_{\mathbf{i}} \underbrace{\sum_{n=1}^{N} \frac{1}{n}\left(\begin{array}{c}
N \\
n
\end{array}\right) P_{\mathbf{i}}^{n}\left(1-P_{\mathbf{i}}\right)^{N-n}}_{\frac{1}{N P_{\mathbf{i}}}+O\left(\frac{1}{N^{2}}\right)} \\
& =2 C^{2} \delta_{2}^{2}+\frac{\overline{\sigma^{2}}}{N} \underbrace{2 C^{2} \sum_{\mathbf{i}} \frac{1}{P_{\mathbf{i}}}}_{C_{1}}+O\left(\frac{1}{N^{2}}\right) \text {. }
\end{aligned}
$$

(a) follows from the continuous compatibility assumption. (b) follows because the noise is chosen independently of the inputs. Choosing $\delta_{1}$ such that $c_{1} \delta_{2}+2 C^{2} \delta_{2}^{2}<\epsilon$ we have

$$
\mathcal{E}_{N}(\sigma) \leq \mathcal{E}_{N}(0)+\frac{C_{1}(\epsilon) \overline{\sigma^{2}}}{N}+\epsilon+O\left(\frac{1}{N^{2}}\right)
$$

We note that it is easy to extend these theorems to the case where the noise variances are drawn from some distribution. By taking the expectation over that distribution, the same result with $\overline{\sigma^{2}}$ being the expected value of the variance parameter is obtained. Note also that the preceding proof is by no means suggesting a method to calculate $C_{1}$. It is simply a means to show its existence. Often, especially when the input distribution is bounded, Corollary B.6 will hold, and it might be possible to estimate these constants experimentally.

One might wonder what would happen if the mean preserving assumption is violated. We note that the only place where this is used is in the evaluation of $T_{1}$. Continuity could still be used, however the difference being that a term of order $\epsilon / \sqrt{N}$ would remain. In other words, one would have $\mathcal{E}_{N}(\sigma) \leq \mathcal{E}_{N}(0)+C^{\prime \prime} \sigma / \sqrt{N}+$ higher order. So if we do not have the mean preserving property, then these methods do not guarantee $1 / N$ convergence of the test error. Using identical methods, one can, however, get the following result using the continuity property alone: $\langle|f-g|\rangle \leq\left\langle\left|f-g_{0}\right|\right\rangle+\left(C^{\prime \prime \prime} \sigma / \sqrt{N}\right)$. This is very similar to Theorem B.5 where one measures test error by the expectation of the magnitude difference as opposed to the squared difference.

We now derive a theorem on the dependence of $\mathcal{E}_{N}(0)$.

Theorem B.9: Let $\mathcal{L}$ be stable. Let the target function $f$ be continuous. Let the probability measure on the input space have compact support. Then $\forall \epsilon>0, \exists C_{2}>0$ such that using $\mathcal{L}$ it is at least possible to attain a noiseless test error bounded by

$$
\mathcal{E}_{N}(0)<E_{0}+\frac{C_{2}}{N}+\epsilon+o\left(\frac{1}{N}\right) .
$$

Corollary B.10: If $C_{2} \leq C_{2}^{\prime} \forall \epsilon$ then $\mathcal{E}_{N}(0) \leq E_{0}+\frac{C_{2}^{\prime}}{N}+$ $o\left(\frac{1}{N}\right)$ where $E_{0}=\lim _{N \rightarrow \infty} \mathcal{E}_{N}(0)$.

Before we proceed to the proof of the theorem, the following lemma is needed.

Lemma B.11: Let $N$ balls be independently be distributed into $r$ cells according to the probabilities $p_{1} \ldots p_{r}$. Then for every $m>0, \exists A_{m}$ such that the probability $q$ that at least one cell is empty is bounded by

$$
q \leq \frac{A_{m}}{N^{m}}
$$

Proof:

$$
\begin{aligned}
q & =\operatorname{Pr}\left[\cup \text { cell }_{i} \text { is empty }\right] \\
& \leq \sum_{i} \operatorname{Pr}\left[\operatorname{cell}_{i} \text { is empty }\right]=\sum_{i}\left(1-p_{i}\right)^{N} \\
& \leq r\left(1-\min _{i} p_{i}\right)^{N} \leq \frac{A_{m}}{N^{m}}
\end{aligned}
$$

choosing $A_{m} \geq r(-m / \ln (a))^{m}$, where $a=1-\min p_{i}$.

Proof of Theorem B.9: Let $\mathcal{X}, \mathcal{S}, \delta_{1}, \delta_{2}, C_{\mathbf{i}}, P_{\mathbf{i}}^{i}, N_{\mathbf{i}}$ be as in the proof of Theorem B.2. We only consider those cubes with $P_{\mathbf{i}}>0$.

Suppose that we have an infinite noiseless data set, $\mathcal{D}_{\infty}$. For all $\mathbf{i}$, let $\bar{y}_{\mathbf{i}}=\langle f(\mathbf{x})\rangle_{\mathbf{x} \mid \mathbf{x} \in C_{\mathbf{i}}}$ and let $\mu_{\mathbf{i}}=\frac{1}{N_{\mathbf{i}}} \sum_{\mathbf{x}_{j} \in C_{\mathbf{i}}} y_{j}$ if $C_{\mathbf{i}}$ is nonempty, else $\mu_{\mathbf{i}}=0$. Construct two data sets from the infinite one, $\mathcal{D}_{1}$ and $\mathcal{D}_{2}$, by replacing all the $y$ 's in $C_{\mathbf{i}}$ by $\bar{y}_{\mathbf{i}}$, and $\mu_{\mathbf{i}}$ respectively. $\mathcal{D}_{1}$ does not depend on $\mathcal{D}_{N}$ and $\mathcal{D}_{2}$ can be obtained from $\mathcal{D}_{N} \cdot \mathcal{D}_{\infty}$ and $\mathcal{D}_{1}$ are close data sets because for $\mathrm{x} \in C_{\mathbf{i}}$

$$
\begin{aligned}
\left|f(\mathbf{x})-\langle f(\mathbf{y})\rangle_{\mathbf{y} \mid \mathbf{y} \in C_{\mathbf{i}}}\right| & =\left|\langle f(\mathbf{x})-f(\mathbf{y})\rangle_{\mathbf{y} \mid \mathbf{y} \in C_{\mathbf{i}}}\right| \\
& \leq\langle|f(\mathbf{x})-f(\mathbf{y})|\rangle_{\mathbf{y} \mid \mathbf{y} \in C_{\mathbf{i}}} \leq \delta_{2} .
\end{aligned}
$$

Therefore, by continuous compatibility $\left\langle\left(g_{\infty}-g_{1}\right)^{2}\right\rangle \leq$ $C^{2} \delta_{2}^{2}$. Define $\epsilon_{\mathbf{i}}$ by $\mu_{\mathbf{i}}=\bar{y}_{\mathbf{i}}+\epsilon_{\mathbf{i}}$. Then $\left\langle\epsilon_{\mathbf{i}}\right\rangle_{\mathcal{D}_{N}}=0$ for all nonempty $C_{\mathbf{i}}$. Let $g_{\infty}, g_{1}$ and $g_{2}$ be $\mathcal{A}\left(\mathcal{D}_{\infty}\right), \mathcal{A}\left(\mathcal{D}_{1}\right)$ and $\mathcal{A}\left(\mathcal{D}_{2}\right)$, respectively. Since we can construct $\mathcal{D}_{2}$, using $\mathcal{L}$ we can at least obtain a test error given by

$$
\begin{aligned}
& \mathcal{E}_{N}(0) \leq \overbrace{\left\langle\left(f-g_{\infty}\right)^{2}\right\rangle}^{E_{0}}+\overbrace{\left\langle\left(g_{\infty}-g_{1}\right)^{2}\right\rangle}^{\leq C^{2} \delta_{2}^{2}}+\left\langle\left(g_{1}-g_{2}\right)^{2}\right\rangle \\
& +2 \underbrace{\left|\left\langle\left(f-g_{\infty}\right)\left(g_{\infty}-g_{1}\right)\right\rangle\right|}_{\substack{\left.\leq\left|f-g_{\infty}\right| \max C \varepsilon_{2} \\
\text { (by cont. comp. }\right)}} \\
& +2\left|\left\langle\left(f-g_{\infty}\right)\left(g_{1}-g_{2}\right)\right\rangle\right| \\
& +2\left|\left\langle\left(g_{\infty}-g_{1}\right)\left(g_{1}-g_{2}\right)\right\rangle\right| \text {. }
\end{aligned}
$$

By the mean preserving property, $\left\langle\left(g_{1}-g_{2}\right)\right\rangle_{\mathcal{D}_{N}}=0$. Therefore

$\left|\left\langle\left(f-g_{\infty}\right)\left(g_{1}-g_{2}\right)\right\rangle\right|=\left|\left\langle\left(f-g_{\infty}\right)\left\langle\left(g_{1}-g_{2}\right)\right\rangle_{\mathcal{D}_{N}}\right\rangle_{\mathbf{x}}\right|=0$. 
Similar reasoning shows that $2\left|\left\langle\left(g_{\infty}-g_{1}\right)\left(g_{1}-g_{2}\right)\right\rangle\right|=0$. Let $Q$ be the probability that at least one cell is empty. For the final term we have

$$
\begin{aligned}
\left\langle\left(g_{1}-g_{2}\right)^{2}\right\rangle & \stackrel{(a)}{\leq}(1-Q) C^{2}\left\langle\sum_{\mathbf{i}} \epsilon_{\mathbf{i}}^{2}\right\rangle_{\mathcal{D}_{N} \mid \forall \mathbf{i}, N_{\mathbf{i}}>0} \\
& +4 Q C^{2}|f|_{\max }^{2} \\
& \leq C^{2}\left\langle\left\langle\sum_{\mathbf{i}} \epsilon_{\mathbf{i}}^{2}\right\rangle_{y_{\mathbf{i}} \mid N_{\mathbf{i}}>0}\right\rangle_{N_{\mathbf{i}}>0}+c_{1} Q \\
& \leq C^{2}\left\langle\sum_{\mathbf{i}} \frac{\sigma_{\mathbf{i}}^{2}}{N_{\mathbf{i}}}\right\rangle_{N_{\mathbf{i}}>0}+c_{1} Q \\
& \stackrel{\text { (b) }}{\leq} \frac{1}{N} \underbrace{C^{2} \sum_{\mathbf{i}} \frac{\sigma_{\mathbf{i}}^{2}}{P_{\mathbf{i}}}}_{C_{2}} x+o\left(\frac{1}{N}\right)
\end{aligned}
$$

where $\sigma_{\mathbf{i}}^{2}=\operatorname{Var}\left(y_{\mathbf{i}} \mid y \in C_{\mathbf{i}}\right)$. (a) follows by continuous compatibility because with probability $1-Q$ the data sets are at most $\epsilon_{\mathbf{i}}$ apart and $\sum_{\mathbf{i}} \epsilon_{\mathbf{i}}^{2} \geq \max _{i} \epsilon_{\mathbf{i}}^{2}$, and with probability $Q$ they are at most $2|f|_{\max }$ apart. (b) follows because $\left\langle 1 / N_{\mathbf{i}}\right\rangle=1 /\left(N P_{\mathbf{i}}\right)+o(1 / N)$, and Lemma B.11 can be used to yield $Q=o(1 / N)$. Finally we have

$$
\mathcal{E}_{N}(0) \leq E_{0}+C^{2} \delta_{2}^{2}+2\left|f-g_{\infty}\right|_{\max } \delta_{2}+\frac{C_{2}}{N}+o\left(\frac{1}{N}\right) .
$$

Choosing $\delta_{2}$ small enough, we have the theorem because $\left|f-g_{\infty}\right|$ is bounded on the compact support $\mathcal{X}$.

\section{ACKNOWLEDGMENT}

The authors would like to thank A. Atiya, J. Sill, Z. Cataltepe, and X. Song for their helpful comments.

\section{REFERENCES}

[1] B. Malkiel, A Random Walk Down Wall Street. New York: Norton, 1985

[2] F. Black and M. S. Scholes, "The pricing of options and corporate liabilities," J. Political Econ., vol. 3, pp. 637-654, 1973.

[3] D. Montgomery, L. Johnson, and J. Gardiner, Forecasting and Time Series Analysis. New York: McGraw-Hill, 1990.

[4] Y. S. Abu-Mostafa and A. F. Atiya, "Introduction to financial forecasting," Appl. Intell., vol. 6, pp. 205-213, 1996.

[5] R. R. Trippi and E. Turban, Neural Networks in Finance and Investing. Chicago: Probos, 1993.

[6] H. White, "Economic prediction using neural networks: The case of IBM daily returns," in Proc. IEEE Int. Conf. Neural Networks, 1988, vol. 2, pp. 451-458.

[7] Y. S. Abu-Mostafa, "Learning from hints," J. Complexity, vol. 10, pp. 165-178, 1994

[8] S. Geman, E. Bienenstock, and R. Doursat, "Neural networks and the bias-variance dilemma," Neural Computation, vol. 4, pp. 1-58, 1992.

[9] N. Murata, S. Yoshizawa, and S. Amari, "Learning curves, model selection and complexity of neural networks," in $A d$ vances in Neural Information Processing Systems, vol. 5, S. J. Hanson, J. D. Cowan, and C. L. Giles, Eds. San Mateo, CA: Morgan Kaufmann, 1993, pp. 607-614.

[10] J. Shao and D. Tu, The Jackknife and the Bootstrap. New York: Springer-Verlag, 1996.
[11] J. E. Moody, "The effective number of parameters: An analysis of generalization and regularization in nonlinear learning systems," in Advances in Neural Information Processing Systems (NIPS), vol. 4, J. E. Moody, S. J. Hanson, and R. P. Lippmann, Eds. 1991, pp. 847-854.

[12] D. Plaut, S. Nowlan, and G. Hinton, "Experiments on learning by backpropagation," Carnegie Mellon Univ., Pittsburgh, PA, Tech. Rep. CMU-CS-86-126, 1986.

[13] S. Amari, N. Murata, K. R. Müller, and H. Yang, "Asymptotic statistical theory of overtraining and cross validation," IEEE Trans. Neural Networks, vol. 8, pp. 985-996, Sept. 1997.

[14] S. Amari and N. Murata, "Statistical theory of learning curves under entropic loss criterion," Neural Computation, vol. 5, no. 1, pp. 140-153, 1993.

[15] J. Hertz, A. Krogh, and R. Palmer, Introduction to the Theory of Neural Computation. Reading, MA: Addison-Wesley, 1991.

[16] A. Krogh, "Learning with noise ins linear perceptron," J. Phys. A, vol. 25, pp. 119-1133, 1992.

[17] G. Leich and J. E. Tanner, "Economic forecast evaluation: Profit versus the conventional error measures," Amer. Econ. Rev., vol. 81 , pp. $580-590,1991$

[18] R. J. Shiller, Market Volatility. Cambridge, MA: MIT, 1993.

[19] V. N. Vapnik and A. Chervonenkis, "On the uniform convergence of relative frequencies of events to their probabilities," Theory of Probability and Its Applications, vol. 16, pp. 264-280, 1971.

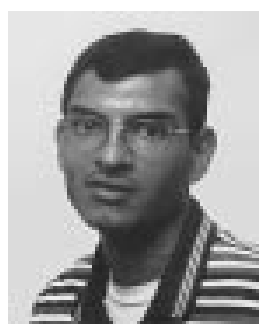

Malik Magdon-Ismail received the B.S. degree in physics from Yale University, New Haven, CT, in 1993, and the M.S. degree in physics and the Ph.D. in electrical engineering from California Institute of Technology (Caltech), Pasadena, in 1995 and 1998, respectively.

He is currently a Postdoctoral Fellow with the Learning Systems Group at Caltech. His research interests include learning in the presence of noise, density estimation, and computational finance.

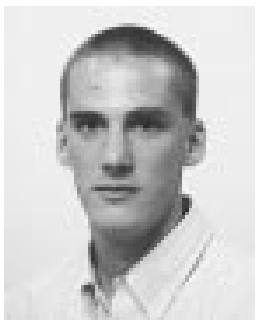

Alexander Nicholson received the B.A.Sc. degree in engineering science from the University of Toronto, Ont., Canada in 1996. He is a graduate student at the California Institute of Technology (Caltech), Pasadena, pursuing the $\mathrm{Ph} . \mathrm{D}$. degree in computer science.

$\mathrm{He}$ is a member of the Learning Systems Group at Caltech. His graduate research focuses on generalization in learning systems and the application of learning algorithms to reconfigurable hardware.

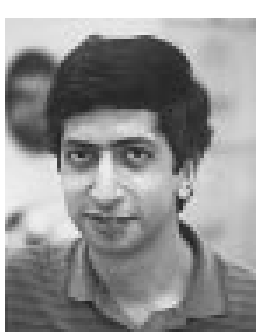

Yaser S. Abu-Mostafa received the B.Sc. degree from Cairo University, Egypt, in 1979 the M.S.E.E. degree from Georgia Institute of Technology, Atlanta, in 1981 and the Ph.D. degree from California Institute of Technology, Pasadena, in 1983

He is currently a Professor of Electrical Engineering and Computer Science at the California Institute of Technology where he also heads the Learning Systems Group. He is the Chairman of the International Conference on Computation Finance (CF'99), and he is a Technical Consultant for a number of financial companies. He has published widely in the areas of learning theory, neural networks, pattern recognition, information theory, and computational complexity. 